\title{
COHERENCY CONDITIONS IN SIMULTANEOUS LINEAR EQUATION MODELS WITH ENDOGENOUS \\ SWITCHING REGIMES
}

C. Gourieroux

J.J. Laffont

A. Monfort

Working Paper No. 343

\section{NATIONAL BUREAU OF ECONOMIC RESEARCH 1050 Massachusetts Avenue Cambridge MA 02138}

May 1979

\begin{abstract}
We greatly benefited from discussions with G. Laroque. The research reported here has been supported, in part, by NSF Grant SOC 78-06162 to the National Bureau of Economic Research and is part of the NBER's research program in Economic Fluctuations. Any opinions expressed are solely those of the authors.
\end{abstract}




\title{
Coherency Conditions in Simultaneous Linear Equation Models
} with Endogenous Switching Regimes

\section{SUMMARY}

In modelling disequilibrium macroeconomic systems which one would want to subject to econometric estimation one typically faces the problem of whether the structural model can determine a unique equilibrium. The problem inherits a special form because the regimes in which the equilibria can lie are each linear. By placing restrictions on the parameters that insure the uniqueness of such a solution for each value of the exogenous and random variables, we can improve the estimation procedure.

This paper provides necessary and sufficient conditions for uniqueness --or "coherency." These conditions are applied to a variety of models that have been prominent in the literature on econometrics with "switching regimes" such as those of self-selectivity (Maddala), simultaneous equation tobit and probit (Amemiya, Schmidt) and multi-market macroeconomic disequilibrium (Gourieroux, Laffont and Monfort).

\author{
C. Gourieroux \\ Ecole Nationale de la Statistique \\ et de 1 'Administration Economique \\ J.J. Laffont \\ Université de Toulouse and Ecole \\ Polytechnique \\ A. Monfort \\ Ecole Nationale de la Statistique \\ et de 1 'Administration Economique
}

Address communications to: J.J. Laffont, Laboratoire d'Econometrie de l'Ecole Polytechnique, 17 Rue Descartes, 75005 Paris, France. 
Let us consider a general econometric model defined as follows :

$$
g\left(y_{t}, x_{t}, u_{t}, \theta\right)=0 \quad t=1, \ldots, T
$$

where $y_{t}$ is a vector of endogenous variables, $x_{t}$ is a vector of exogenous variables, $u_{t}$ is a vector of perturbations, $\theta$ is a vector of unknown parameters and $g$ is a known vector function.

If this model is to be used for econometric purposes, it must associate a unique value of $y_{t}$ with any admissible value of $x_{t}, u_{t}$ and $\theta$; in other words, the model must have a well-defined reduced form :

$$
y_{t}=h\left(x_{t}, u_{t}, \theta\right)
$$

In the sequel we shall call "coherency conditions" the conditions on the parameters $\theta$ that insure this property.

This coherency problem must be distinguished from the identifiability problem which can be meaningfully stated only for a model satisfying the coherency property. Indeed the identifiability of a model, which is the uniqueness of the parameters of the model given the distribution of the observable variables, presupposes the existence of a well-defined distribution for the endogenous variables. For instance, in the general linear model :

$$
B y_{t}+C x_{t}=u_{t}
$$

the coherency condition reduces to the invertibility of $B$ (i.e. $\operatorname{det} B \neq 0$ ) whereas the first-order identifiability amounts to the uniqueness of $B$ and $C$ given $B^{-1} C$. 
In non linear models the issue of coherency is usually incompletely dealt with by assuming the differentiability of $g$ and requiring a non vanishing Jacobian $\left|\frac{\partial g}{\partial y_{t}}\right|$. The latter condition insures only locally the obtainability of a reduced form. Moreover, the constraints implied on the parameters are never spelled out.

In this paper we provide an explicit solution of the coherency problem for a particular class of non linear models, namely the class of simultaneous linear equation models with endogenously switching regimes. In this case $g$ is piecewise linear in $y_{t}$ and the coherency property is the invertibility of this piecewise linear mapping.

Recently the literature has offered a large variety of such models, in particular the self-selectivity models (e.g. MADDALA [ 9 ]), the simultaneous equation Probit and Tobit models (e.g. AMEMIYA [1], SCHMIDT [13]) or the simultaneous equation disequilibrium models (e.g. GOURIEROUX, LAFFONT and MONFORT [3]).

In the next four sections we provide necessary and sufficient conditions of coherency for the general piecewise linear model under four different sets of assumptions. Each case is illustrated by various examples borrowed from the literature. 
1- TYPE 1 MODELS : CONTINUOUS PIECENISE LINEAR MAPPINGS ON CONES DEFINED BY ENDOGENOUS VARTABLES.

Consider the Euclidian space, $\mathbb{R}^{\mathfrak{n}}$, and consider $\mathrm{n}$ independent linear forms, $a_{1}, \ldots, a_{n}$, defined on $\mathbb{R}^{n}$. For each subset $I$ of the set $\{1,2, \ldots, n\}$, let $C_{I}$ be the cone defined by :

$$
\begin{aligned}
& c_{I}=\left\{x \mid x \in \mathbb{R}^{n}, \quad a_{i} x \geqslant 0 \quad \text { if } i \in I\right. \text { and } \\
& \left.a_{i} x<0 \quad \text { if } i \notin I\right\}
\end{aligned}
$$

There are $2^{\mathrm{n}}$ such cones ; they coincide with the orthants of $\mathbb{R}^{n}$ if each linear form $a_{i}$ is the $i^{\text {th }}$ coordinate projection function : $a_{i} x=x_{i}$.

Let us associate with each cone an invertible linear mapping, $A_{I}$, from $\mathbb{R}^{n}$ into $\mathbb{R}^{n}$. Then, consider the mapping $\left.f=\sum_{I} A_{I}\right]_{C} C_{I}$ where

$$
\begin{aligned}
\nabla_{C_{I}(x)} & =1 \quad \text { if } x \in C_{I} \\
& =0 \quad \text { if } x \notin C_{I}
\end{aligned}
$$

The mapping $f$ is therefore a piecewise linear mapping from $\mathbb{R}^{\mathrm{n}}$ into $\mathbb{R}^{\mathrm{n}}$ defined by the linear mapping $\mathrm{A}_{I}$ on each cone $\mathrm{C}_{I}$ with $U_{I} C_{I}=\mathbb{R}^{n}$. Note that the mappings $A_{I}$ need not be different ; in 
that case, the relevant partition of $\mathbb{R}^{\mathbb{n}}$ in cones may have less than $2^{n}$ elements.

We will see in the examples below that the possibility. of having a well-defined reduced form in a model where the structural form is piecewise linear depends on the invertibility of a mapping such as $f$.

In this section we assume, as it is often the case in applications, that the matrices $A_{I}$ are constrained in such a way that the function $f$ is continuous. We can then state our first invertibility theorem $^{1)}$.

Theorem 1:

Suppose that the mapping $f=\sum_{I} A_{I}{ }^{1} C_{I}$ is continuous

from $\mathbb{R}^{\mathbb{n}}$ to $\mathbb{R}^{\mathbb{n}}$. A necessary and sufficient confition for $f$ to be invertible is that all the determinants, $\operatorname{det} A_{I}$,

$I \subset\{1,2, \ldots, \mathrm{n}\}$, have the same sign.

We show below how to use this theorem in examples presented in a very concise form ${ }^{2}$.

1) For expository purposes the proots are gathered in appendices.

2) The reader might find profitable to go through the origiral papers and see how the coherency issue was dealt with in each case. 
Example 1.1.

Consider the following model discussed by LEE [8]

$$
\begin{aligned}
& y_{1 t}=\gamma_{1} y_{2 t}+\delta ; x_{1 t}+u_{1 t} \\
& y_{2 t}=\gamma_{2} y_{1 t}+\delta_{2}^{\prime} x_{2 t}+u_{2 t} \quad \text { if } y_{1 t} \geqslant 0 \\
& =\delta_{2}^{\prime} x_{2 t}+u_{2 t} \quad \text { if } y_{1 t}<0 \\
& t=1, \ldots, T
\end{aligned}
$$

where $\left(y_{1 t}, y_{2 t}\right)$ are endogenous variables, $\left(x_{1 t}, x_{2 t}\right)$ are exogenous variables and $\left(u_{1 t}, u_{2 t}\right)$ are random disturbances.

The problem of the existence of a reduced form for this model is identical to the problem of the invertibility of the mapping from $\left(y_{1 t}, y_{2 t}\right)$ to $\left(\delta_{1}^{\prime} x_{1 t}+u_{1 t}, \delta_{2}^{\prime} x_{2 t}+u_{2 t}\right)$ defined by

$$
\begin{array}{r}
f=\sum_{i=1}^{4} A_{i} \mathbb{1}_{i} \text { and } c_{1}=\left\{\left(y_{1 t}, y_{2 t}\right): y_{1 t} \geqslant 0 \quad y_{2 t} \geqslant 0\right\} \\
c_{2}=\left\{\left(y_{1 t}, y_{2 t}\right): y_{1 t} \geqslant 0 \quad y_{2 t}<0\right\} \\
c_{3}=\left\{\left(y_{1 t}, y_{2 t}\right): y_{1 t}<0 \quad y_{2 t}<0\right\} \\
c_{4}=\left\{\left(y_{1 t}, y_{2 t}\right): y_{1 t}<0, y_{2 t} \geqslant 0\right\} \\
\text { on } c_{1} \cup c_{2}, \quad \text { the mapping is } A_{1}=A_{2}=\left[\begin{array}{rr}
1 & -\gamma_{1} \\
-\gamma_{2} & 1
\end{array}\right]
\end{array}
$$


On $C_{3} \cup C_{4}$, the mapping is $A_{3}=A_{4}=\left[\begin{array}{rr}1 & -\gamma_{1} \\ 0 & 1\end{array}\right]$

The mapping $f$ is clearly continuous since, on the common boundary of the closures of $C_{1} \cup C_{2}$ and $C_{3} \cup C_{4}, A_{1}$ and $A_{3}$ coincide. Indeed for any $a \in \mathbb{R}$

$$
A_{1}\left[\begin{array}{l}
0 \\
a
\end{array}\right]=\left[\begin{array}{cc}
-\gamma_{1} & a \\
& \\
a
\end{array}\right]=A_{3}\left[\begin{array}{l}
0 \\
a
\end{array}\right]
$$

From theorem 1, the coherency condition is $\operatorname{det} A_{1} \cdot \operatorname{det} A_{3}>0$, or $1-\gamma_{1} \gamma_{2}>0$

\section{Example 1.2.}

Consider the following demand and supply disequilibrium model (LAFFONT and MONFORT [7])

$$
\begin{aligned}
& D_{t}=\gamma_{1} P_{t}+\delta_{1}^{\prime} x_{1 t}+u_{1 t} \\
& S_{t}=\gamma_{2} P_{t}+\delta_{2}^{\prime} x_{2 t}+u_{2 t}
\end{aligned}
$$

where $P_{t}$ is the price of the commodity and $\left(x_{1 t}, x_{2 t}\right)$ are exogenous variables. The exchanged quantity is

$$
Q_{t}=\min \left(D_{t}, S_{t}\right)
$$


and the price dynamics is defined by

$$
\begin{aligned}
& P_{t}-P_{t-1}=\Delta P_{t}=\lambda_{1}\left(D_{t}-S_{t}\right), \lambda_{1}>0, \text { if } D_{t} \geqslant s_{t} \\
& P_{t}-P_{t-1}=\Delta P_{t}=\lambda_{2}\left(D_{t}-S_{t}\right), \lambda_{2}>0, \text { if } D_{t}<s_{t}
\end{aligned}
$$

The mapping from endogenous variables $\left(D_{t}, S_{t}\right)$ to the random disturbances and exogenous variables $\left(u_{1 t}+\delta_{1}^{\prime} x_{1 t}+\gamma_{1} P_{t-1}\right.$, $\left.u_{2 t}+\delta_{2}^{\prime} x_{2 t}+\gamma_{2} P_{t-1}\right)$ can be written

$$
\begin{gathered}
A_{1}\left[\begin{array}{l}
D_{t} \\
s_{t}
\end{array}\right]=\left[\begin{array}{cc}
1-\gamma_{1} \lambda_{1} & \gamma_{1} \lambda_{1} \\
-\gamma_{2} \lambda_{1} & 1+\lambda_{1} \gamma_{2}
\end{array}\right]\left[\begin{array}{l}
D_{t} \\
s_{t}
\end{array}\right]=\left[\begin{array}{l}
u_{1 t}+\delta_{1}^{\prime} x_{1 t}+\gamma_{1} P_{t-1} \\
u_{2 t}+\delta_{2}^{\prime} x_{2 t}+\gamma_{2} P_{t-1}
\end{array}\right] \\
\text { if } D_{t}-s_{t} \geqslant 0
\end{gathered}
$$

$$
\begin{gathered}
A_{2}\left[\begin{array}{l}
D_{t} \\
s_{t}
\end{array}\right]=\left[\begin{array}{cc}
1-\gamma_{1} \lambda_{2} & \gamma_{1} \lambda_{2} \\
-\gamma_{2} \lambda_{2} & 1+\lambda_{2} \gamma_{2}
\end{array}\right]\left[\begin{array}{l}
D_{t} \\
s_{t}
\end{array}\right]=\left[\begin{array}{l}
u_{1 t}+\delta_{1}^{\prime} x_{1 t}+\gamma_{1} P_{t-1} \\
u_{2 t}+\delta_{2}^{\prime} x_{2 t}+\gamma_{2} P_{t-1}
\end{array}\right] \\
\text { if } D_{t}-s_{t}<0
\end{gathered}
$$

$A_{1}$ and $A_{2}$ coincide for equilibrium points $\left(D_{t}=S_{t}\right)$.

The coherency condition $\operatorname{det} A_{1} \cdot \operatorname{det} A_{2}>0$ reduces here to

$\left[1+\lambda_{1}\left(\gamma_{2}-\gamma_{1}\right)\right] \cdot\left[1+\lambda_{2}\left(\gamma_{2}-\gamma_{1}\right)\right]>0$

which is in general satisfied in a supply-demand model since $\gamma_{1}<0, \gamma_{2}>0$. 
There is another piecewise linear continuous mapping between $\left(D_{t}, S_{t}\right)$ and the observable variables, namely, $\left(Q_{t}, \Delta P_{t}\right)$. It is defined by the matrices $\left[\begin{array}{cc}0 & 1 \\ \lambda_{1} & -\lambda_{1}\end{array}\right]$ and $\left[\begin{array}{cc}1 & 0 \\ \lambda_{2} & -\lambda_{2}\end{array}\right]$. Applying again theorem 1 we see that the mapping is one to one since $\lambda_{1}>0, \lambda_{2}>0$.

Consequently the mapping from the random disturbances and exogenous variables to the observables is one to one when $\lambda_{1}>0$, $\lambda_{2}>0, \gamma_{1}<0, \gamma_{2}>0$, ensuring a well defined reduced form for this problem.

\section{Example 1.3.}

GOLDFELD and QUANDT [5] have studied a model which can be defined as follows :

$$
\begin{aligned}
& R_{t}=\delta_{1}^{\prime} x_{1 t}+u_{1 t} \\
& \Sigma_{t}=B_{2} R_{t}+\gamma_{2} P_{t}+\delta_{2}^{\prime} x_{2 t}+u_{2 t}
\end{aligned}
$$

where $R_{t}$ defines the crop of the comodity, $\Sigma_{t}$ the desired harvest; $s_{t}=\min \left(R_{t}, \Sigma_{t}\right)$ is the actual supply, i.e. the actual harvest.

The demand is defined in the form of an inverse demand function

$$
P_{t}=Y_{3} D_{t}+\delta_{3}^{\prime} X_{3 t}+u_{3 t}
$$

and the price adjusts to equate demand and actual supply $\left(D_{t}=S_{t}\right)$. 
This model can be rewritten as a two regime model :

$$
\begin{aligned}
& \text { Regime } 1 \quad\left(R_{t}<\Sigma_{t}\right) \\
& {\left[\begin{array}{ccc}
1 & 0 & 0 \\
-B_{2} & 1 & -\gamma_{2} \\
-\gamma_{3} & 0 & 1
\end{array}\right]\left[\begin{array}{c}
R_{t} \\
\Sigma_{t} \\
P_{t}
\end{array}\right]=\left[\begin{array}{c}
\delta_{1}^{\prime} x_{1 t}+u_{1 t} \\
\delta_{2}^{\prime} x_{2 t}+u_{2 t} \\
\delta_{3}^{\prime} x_{3 t}+u_{3 t}
\end{array}\right]} \\
& \text { Regime } 2\left(R_{t}>\Sigma_{t}\right) \\
& {\left[\begin{array}{ccc}
1 & 0 & 0 \\
-B_{2} & 1 & -\gamma_{2} \\
0 & -\gamma_{3} & 1
\end{array}\right]\left[\begin{array}{c}
R_{t} \\
\Sigma_{t} \\
P_{t}
\end{array}\right]=\left[\begin{array}{c}
\delta_{1}^{\prime} x_{1 t}+u_{1 t} \\
\delta_{2}^{\prime} x_{2 t}+u_{2 t} \\
\delta_{3}^{\prime} x_{3 t}+u_{3 t}
\end{array}\right]}
\end{aligned}
$$

The coherency condition is therefore $\left(1-\gamma_{3} \gamma_{2}\right)>0$ which is true if $\gamma_{3}<0, \gamma_{2}>0$ as may be expected (see footnote 6 in GULDFELD and QUANDT [5]).

\section{Example 1.4.}

The generalization of the Tobit model leads to the following type of sys tem (see AMEMIYA [ 1]).

$$
\begin{aligned}
& y_{1 t}=\gamma_{1} y_{2 t}+\delta_{1}^{\prime} x_{1 t}+u_{1 t} \text { if } \quad \gamma_{1} y_{2 t}+\delta_{1}^{\prime} x_{1 t}+u_{1 t} \geqslant 0 \\
& =0 \\
& \text { if } \quad \gamma_{1} y_{2 t}+\delta_{1}^{\prime} x_{1 t}+u_{1 t}<0
\end{aligned}
$$




$$
\begin{gathered}
y_{2 t}=\gamma_{2} y_{1 t}+\delta_{2}^{\prime} x_{2 t}+u_{2 t} \quad \text { if } \gamma_{2} y_{1 t}+\delta_{2}^{\prime} x_{2 t}+u_{2 t} \geqslant 0 \\
=0 \quad \text { if } \gamma_{2} y_{1 t}+\delta_{2}^{\prime} x_{2 t}+u_{2 t}<0 \\
\text { Setting } Y_{1 t}=\gamma_{1} y_{2 t}+\delta_{1}^{\prime} x_{1 t}+u_{1 t}, \\
Y_{2 t}=\gamma_{2} y_{1 t}+\delta_{2}^{\prime} x_{2 t}+u_{2 t},
\end{gathered}
$$

we observe that $y_{1 t}=\sup \left(Y_{1 t}, 0\right)$ and $y_{2 t}=\sup \left(Y_{2 t}, 0\right)$ and that the system can be rewritten, in terms of $\left(Y_{1 t}, Y_{2 t}\right)$, as:

$$
\begin{aligned}
& Y_{1 t}=\gamma_{1} \text { sup }\left(Y_{2 t}, 0\right)+\delta_{1}^{\prime} x_{1 t}+u_{1 t} \\
& Y_{2 t}=\gamma_{2} \text { sup }\left(Y_{1 t}, 0\right)+\delta_{2}^{\prime} x_{2 t}+u_{2 t} \\
& \text { The four relevant matrices are therefore } \\
& {\left[\begin{array}{cc}
1 & -\gamma_{1} \\
-\gamma_{2} & 1
\end{array}\right] ;\left[\begin{array}{ll}
1 & -\gamma_{1} \\
0 & 1
\end{array}\right] ;\left[\begin{array}{ll}
1 & 0 \\
0 & 1
\end{array}\right] ;\left[\begin{array}{ll}
1 & 0 \\
-\gamma_{2} & 1
\end{array}\right]} \\
& \text { and the coherency condition reduces to } \\
& \text { l }-\gamma_{1} \gamma_{2}>0
\end{aligned}
$$

Indeed, AMEMIYA [1] obtained this condition by applying directly a theorem which is closed related to the SAMELSON, THRALL and WESLER theorem [12] that we use in proving our results (see appendix 1). 
Example 1.5.

To deal with the case of two markets in a disequilibrium framework, QUANDT [11] has proposed the following model where prices are exogenous :

$$
\begin{aligned}
& D_{1 t}=\gamma_{1} Q_{2 t}+\delta_{1}^{\prime} x_{1 t}+u_{1 t} \\
& S_{1 t}=\gamma_{2} Q_{2 t}+\delta_{2}^{\prime} x_{2 t}+u_{2 t} \\
& D_{2 t}=\gamma_{3} Q_{1 t}+\delta_{3}^{\prime} x_{3 t}+u_{3 t} \\
& S_{2 t}=\gamma_{4} Q_{1 t}+\delta_{4}^{\prime} x_{4 t}+u_{4 t}
\end{aligned}
$$

and where the exchanged quantities $\left(Q_{1 t}, Q_{2 t}\right)$ are defined by

$$
\begin{aligned}
& Q_{1 t}=\min \left(D_{1 t}, S_{1 t}\right) \\
& Q_{2 t}=\min \left(D_{2 t}, S_{2 t}\right)
\end{aligned}
$$

$$
\begin{aligned}
& \text { The mapping from ( } \left.D_{1 t}, s_{1 t}, D_{2 t}, s_{2 t}\right) \text { to } \\
& \left(u_{1 t}+\delta_{1}^{\prime} x_{1 t}, u_{2 t}+\delta_{2}^{\prime} x_{2 t}, u_{3 t}+\delta_{3}^{\prime} x_{3 t}, u_{4 t}+\delta_{4}^{\prime} x_{4 t}\right) \text { is } \\
& f=\sum_{i=1}^{4} A_{i}{ }^{1} C_{i}
\end{aligned}
$$


$-12-$

$$
\begin{aligned}
& c_{1}=\left\{D_{1 t}, s_{1 t}, D_{2 t}, s_{2 t} / D_{1 t}-s_{1 t} \geqslant 0, D_{2 t}-s_{2 t} \geqslant 0\right\} \\
& c_{2}=\left\{D_{1 t}, s_{1 t}, D_{2 t}, s_{2 t} / D_{1 t}-s_{1 t} \geqslant 0, D_{2 t}-s_{2 t}<0\right\} \\
& c_{3}=\left\{D_{1 t}, s_{1 t}, D_{2 t}, s_{2 t} / D_{1 t}-s_{1 t}<0, D_{2 t}-s_{2 t}<0\right\} \\
& c_{4}=\left\{D_{1 t}, s_{1 t}, D_{2 t}, s_{2 t} / D_{1 t}-s_{1 t}<0, D_{2 t}-s_{2 t} \geqslant 0\right\}
\end{aligned}
$$

and

$$
\begin{aligned}
& A_{1}=\left[\begin{array}{cccc}
1 & 0 & 0 & -\gamma_{1} \\
0 & 1 & 0 & -\gamma_{2} \\
0 & -\gamma_{3} & 1 & 0 \\
0 & -\gamma_{4} & 0 & 1
\end{array}\right] ; A_{2}=\left[\begin{array}{cccc}
1 & 0 & -\gamma_{1} & 0 \\
0 & 1 & -\gamma_{2} & 0 \\
0 & -\gamma_{3} & 1 & 0 \\
0 & -\gamma_{4} & 0 & 1
\end{array}\right]
\end{aligned}
$$

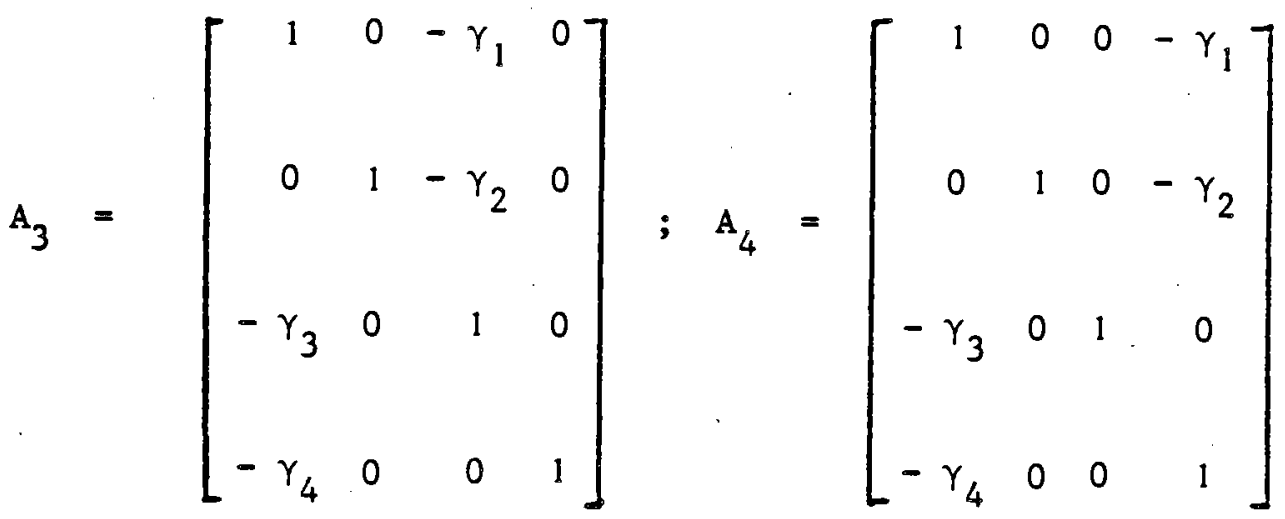


It is easy to verify that the mappings $\left(A_{i}\right)$ coincide on the common boundaries of the cones $\left(C_{i}\right)$ on which they are relevant. Therefore theorem 1 can be applied; the coherency conditions are that the determinants $\left(1-\gamma_{2} \gamma_{4}\right),\left(1-\gamma_{2} \gamma_{3}\right),\left(1-\gamma_{1} \gamma_{3}\right)$, $\left(1-\gamma_{1} \gamma_{4}\right)$ must be of the same sign.

It is worth pointing out the difference which exists between the coherency conditions and the stability conditions of the "natural" dynamic process associated with the above system.

Consider one of the four cases defined above, say case 1 , where $D_{1 t} \geqslant S_{1 t}$ and $D_{2 t} \geqslant S_{2 t}$. The exchanged quantities are defined by

$$
\begin{aligned}
& Q_{1 t}=s_{1 t}=\gamma_{2} Q_{2 t}+\delta_{2}^{\prime} x_{2 t}+u_{2 t} \\
& Q_{2 t}=s_{2 t}=\gamma_{4} Q_{1 t}+\delta_{4}^{\prime} x_{4 t}+u_{4 t}
\end{aligned}
$$

By analogy with the Walrasian adjustment process we can define a quantity adjustment process as follows

$$
\begin{aligned}
& \dot{Q}_{1}=F\left(\gamma_{2} Q_{2}+\delta_{2}^{\prime} x_{2}+u_{2}-Q_{1}\right), F(0)=0, F^{\prime}>0 \\
& \dot{Q}_{2}=G\left(\gamma_{4} Q_{1}+\delta_{4}^{\prime} x_{4}+u_{4}-Q_{2}\right), G(0)=0, G^{\prime}>0
\end{aligned}
$$


designed to converge to the (fixed price) equilibrium of type 1 . The linearization of this system around the equilibrium $\left(Q_{1}^{e}, Q_{2}^{e}\right)$ yields :

$$
\left[\begin{array}{c}
\dot{Q}_{1} \\
\dot{Q}_{2}
\end{array}\right]=\left[\begin{array}{cc}
-F^{\prime} & Y_{2} F^{\prime} \\
Y_{4} G^{\prime} & -G^{\prime}
\end{array}\right]\left[\begin{array}{c}
Q_{1}-Q_{1}^{e} \\
Q_{2}-Q_{2}^{e}
\end{array}\right]
$$

Therefore, the adjustment process is locally stable if and only if the real parts of the characteristic values of the matrix $\left[\begin{array}{cc}-F^{\prime} & \gamma_{2} F^{\prime} \\ \gamma_{4} G^{\prime} & -G^{\prime}\end{array}\right]$ are negative. The sum of the characteristic values is negative, since the trace $-\left(F^{\prime}+G^{\prime}\right)$ is negative. The product of the characteristic values equal to the determinant $F^{\prime} G^{\prime}\left(1-\gamma_{2} \gamma_{4}\right)$ must therefore be positive in order that the real parts of the characteristic values be negative (actually the characteristic values are real in this particular case). The local stability of the quantity adjustment processes in every regime is therefore equivalent to the coherency conditions.

The generalization of this approach to the $n$-market case is straightforward. The model can be written as ${ }^{3}$ ):

3) See GOURIEROUX, LAFFONT and HONFORT [3] for a precise definition of the notion of eófective demand which is implicit in this model. 


$$
\begin{aligned}
& D_{1 t}=\sum_{j \neq 1} \gamma_{1 j} Q_{j t}+\delta_{1}^{\prime} x_{1 t}+u_{1 t} \\
& S_{1 t}=\sum_{j \neq 1} \gamma_{2 j} Q_{j t}+\delta_{2}^{\prime} x_{2 t}+u_{2 t} \\
& D_{n t}=\sum_{j \neq n} \gamma_{(2 n-1) j} Q_{j t}+\delta_{(2 n-1)}^{\prime} x_{(2 n-1) t}+u_{(2 n-1) t} \\
& S_{n t}=\sum_{j \neq n} \gamma_{(2 n) j} Q_{j t}+\delta_{2 n}^{\prime} x_{(2 n) t}+u_{(2 n) t} \\
& Q_{j t}=\min \left(D_{j t}, S_{j t}\right) \\
& \mathrm{j}=1, \ldots, \mathrm{n}
\end{aligned}
$$

There are $2^{\mathrm{n}}$ regimes according to which markets are constrained on the supply side and which markets are constrained on the demand side.

Suppose for example that in the $k$ first markets the demand is constrained and in the $(n-k)$ next markets the supply is constrained. Then $Q_{j t}=S_{j t}$ for $j=1, \ldots, k$ and $Q_{j t}=D_{j t}$ for $\mathrm{j}=\mathrm{k}+1, \ldots, \mathrm{n}$.

In that regime the system of supply and demand equations can be rewritten : 


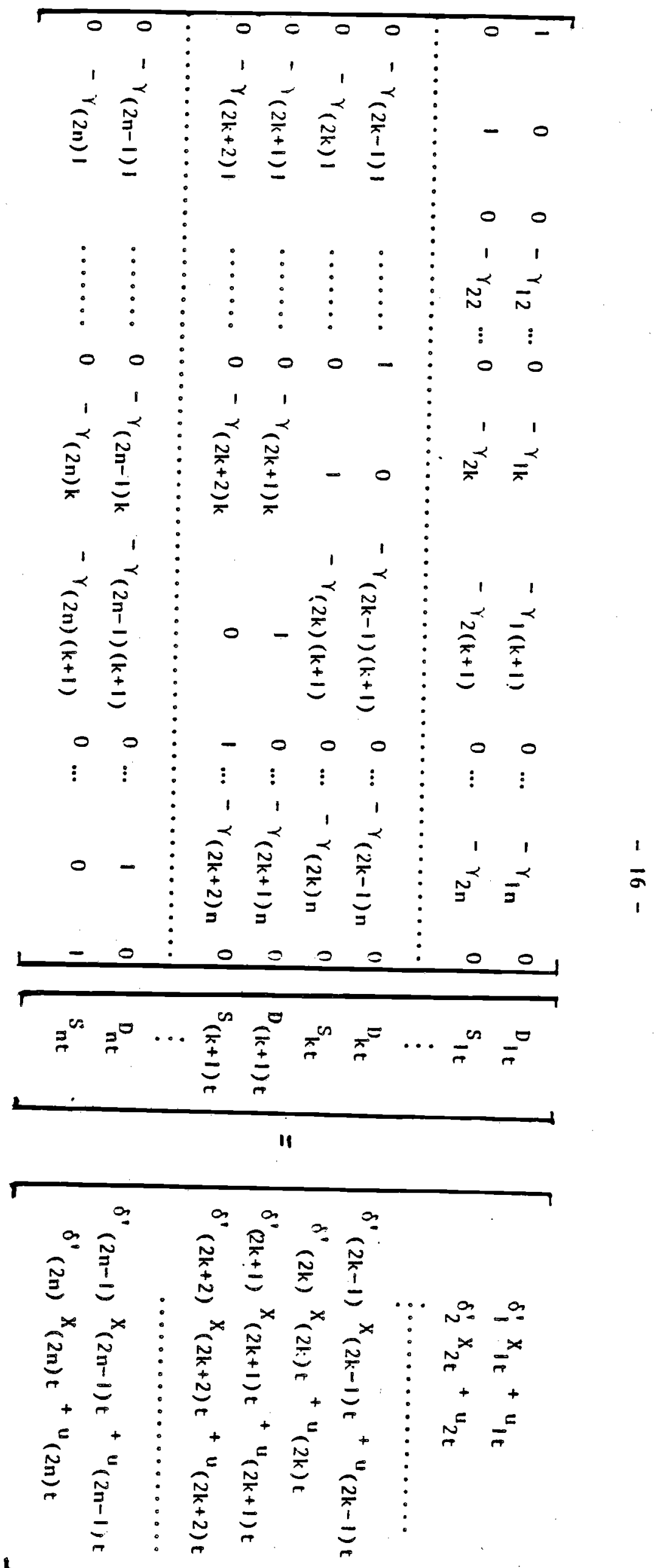


The $\left(Y_{i j}\right)$ are the spillover coefficients. To each regime corresponds a different matrix $\left(A_{I}\right)$ of spillover coefficients. The above mapping is relevant on the cone $\left\{D_{1 t}, s_{1 t}, \ldots, D_{n t}, s_{n t}\right.$ : $D_{1 t}-s_{1 t} \geqslant 0, \ldots, D_{k t}-s_{k t} \geqslant 0, D_{(k+1) t}-s_{(k+1) t}<0, \ldots$, $\left.D_{n t}-s_{n t}<0\right\}$.

The coincidence on the common boundaries of these cones is easily checked. The coherency condition requires therefore that the determinants of all these matrices be of the same sign. This is a quite messy condition. However it is much weaker than a condition of stability of each of these matrices $\left(A_{I}\right)$.

Beyond the $n=2$ case, stability implies coherency but not the converse. Indeed stability requires that the real parts of the characteristic values be negative. Since complex characteristic values always appear in pairs of conjugate values, the determinant which equals the product of characteristic values is, under the stability conditions, of the sign of $(-1)^{n}$. In particular this implies that the determinants are of the same sign in all regimes ${ }^{4}$ ).

4) To prove the existence of a well defined reduced form in this model ITO [6] imposes a condition of diagonal dominance on each matrix $A_{I}$ - This condition implies "stability" of each matrix and therefore coherency. 


\section{2 - TYPE 2 HODELS : PIECEUISE LINEAR :IAPPINGS ON CONES DEFINED BY ENDOGENOUS VARIABLES.}

We will now relax the assumption of continuity that we imposed above on the mapping $f=\sum_{I} A_{I} \mathbb{1}_{C_{I}}$. Indeed, only weaker conditions have to be required along the boundaries of the cones.

Let $a_{1}, \ldots, a_{p}$ be $p(\leqslant n)$ independent linear forms defined, on $\mathbb{R}^{\mathrm{n}}$, and for any subset $I \subset\{1,2, \ldots, \mathrm{p}\}$ let $\mathrm{C}_{I}$ be the cone defined as :

$$
C_{I}=\left\{x \mid x \in \mathbb{R}^{n}, a_{i} x \geqslant 0 \text { if } i \in I \text { and } a_{j} x<0 \text { if } j \notin I\right\}
$$

To each of the $2^{\mathrm{P}}$ cones we associate an invertible linear mapping, $A_{I}$, from $\mathbb{R}^{n}$ into $\mathbb{R}^{n}$.

\section{Theorem 2 :}

If $A_{I}(x)$ is independent of $I$ for any $x$ in $\left\{x \mid x \in \mathbb{R}^{n}\right.$, $\left.a_{j} x=0, j=1, \ldots, p\right\}$ and if, for any $I$ containing $i, A_{I}$ $\left(\left\{x \mid x \in \mathbb{R}^{n}, a_{i} x \geqslant 0\right.\right.$ and $\left.\left.a_{j} x=0, \forall j \neq i\right\}\right)$ is independent of $I$ and,for any $I$ not containing $i, A_{i}\left(\left\{x \mid x \in \mathbb{R}^{n}, a_{i} x<0\right.\right.$ and $\left.\left.a_{j} x=0, \forall j \neq i\right\}\right)$ is independent of $I$, then $f=\sum A_{I} \mathbb{Z}_{I}$ is invertible, if and only if, all the determinants, $\operatorname{det} \mathrm{A}_{I}$, $I \subset\{1,2, \ldots, p\}$ have the same sign. 
The first condition requires that the mappings, $A_{I}$, coincide, point by point, on the intersection of the subspaces defining the cones $C_{I}$; the second condition is a global coincidence of these mappings on appropriate facets of the cones.

\section{Example 2.1.:}

The first example of application of this theorem appeared in GOURIEROUX and alii [3] where it was proved directly for $n=4$, $\mathrm{p}=2$

The structural form of a two market disequilibrium model is obtained, using the Clower effective demand. As in Quandt's model (example 1.5.), four regimes are obtained, according to the signs of the excess demands on the two markets.

For example, in regime 1 where there is excess demand on both markets (the cone $C_{1}$ is defined by $D_{1 t}-s_{1 t} \geqslant 0$, $D_{2 t}-S_{2 t} \geqslant 0$ ), the structural form is:

$$
\begin{aligned}
& Q_{1 t}=s_{1 t}=\gamma_{2} Q_{2 t}+\delta_{2}^{\prime} x_{2 t}+u_{2 t} \\
& Q_{2 t}=s_{2 t}=\gamma_{4} Q_{1 t}+\delta_{4}^{\prime} x_{4 t}+u_{4 t} \\
& D_{1 t}=\frac{1}{1-\gamma_{1} \gamma_{4}}\left[\gamma_{1}\left(\delta_{4}^{\prime} x_{4 t}+u_{4 t}\right)+\delta_{1}^{\prime} x_{1 t}+u_{1 t}\right] \\
& D_{2 t}=\frac{1}{1-\gamma_{2} \gamma_{3}}\left[\gamma_{3}\left(\delta_{2}^{\prime} x_{2 t}+u_{2 t}\right)+\delta_{3}^{\prime} x_{3 t}+u_{3 t}\right]
\end{aligned}
$$


where $D_{1 t}$ and $D_{2 t}$ are the Clower effective demands and $Q_{1 t}$, $Q_{2 t}$ are the exchanged quantities. In this regime the Clower effective demands coincide with the Walrasian demands (see GOURIEROUX and alii [3] for details).

This system can be rewritten as:

$A_{1}\left[\begin{array}{c}D_{1 t} \\ s_{1 t} \\ D_{2 t} \\ s_{2 t}\end{array}\right]=\left[\begin{array}{cccc}1-\gamma_{1} \gamma_{4} & \gamma_{1} \gamma_{4} & 0 & -\gamma_{1} \\ 0 & 1 & 0 & -\gamma_{2} \\ 0 & -\gamma_{3} & 1-\gamma_{2} \gamma_{3} & \gamma_{2} \gamma_{3} \\ 0 & -\gamma_{4} & 0 & 1\end{array}\right]\left[\begin{array}{c}D_{1 t} \\ s_{1 t} \\ D_{2 t} \\ s_{2 t}\end{array}\right]=\left[\begin{array}{c}\delta_{1}^{\prime} x_{1 t}+u_{1 t} \\ \delta_{2}^{\prime} x_{2 t}+u_{2 t} \\ \delta_{3}^{\prime} x_{3 t}+u_{3 t} \\ \delta_{4}^{\prime} x_{4 t}+u_{4 t}\end{array}\right]$

Similarly, one obtains three other mappings :

$$
A_{2}=\left[\begin{array}{cccc}
1 & 0 & -\gamma_{1} & 0 \\
0 & 1 & -\gamma_{2} & 0 \\
0 & -\gamma_{3} & 1 & 0 \\
0 & -\gamma_{4} & 0 & 1
\end{array}\right]
$$




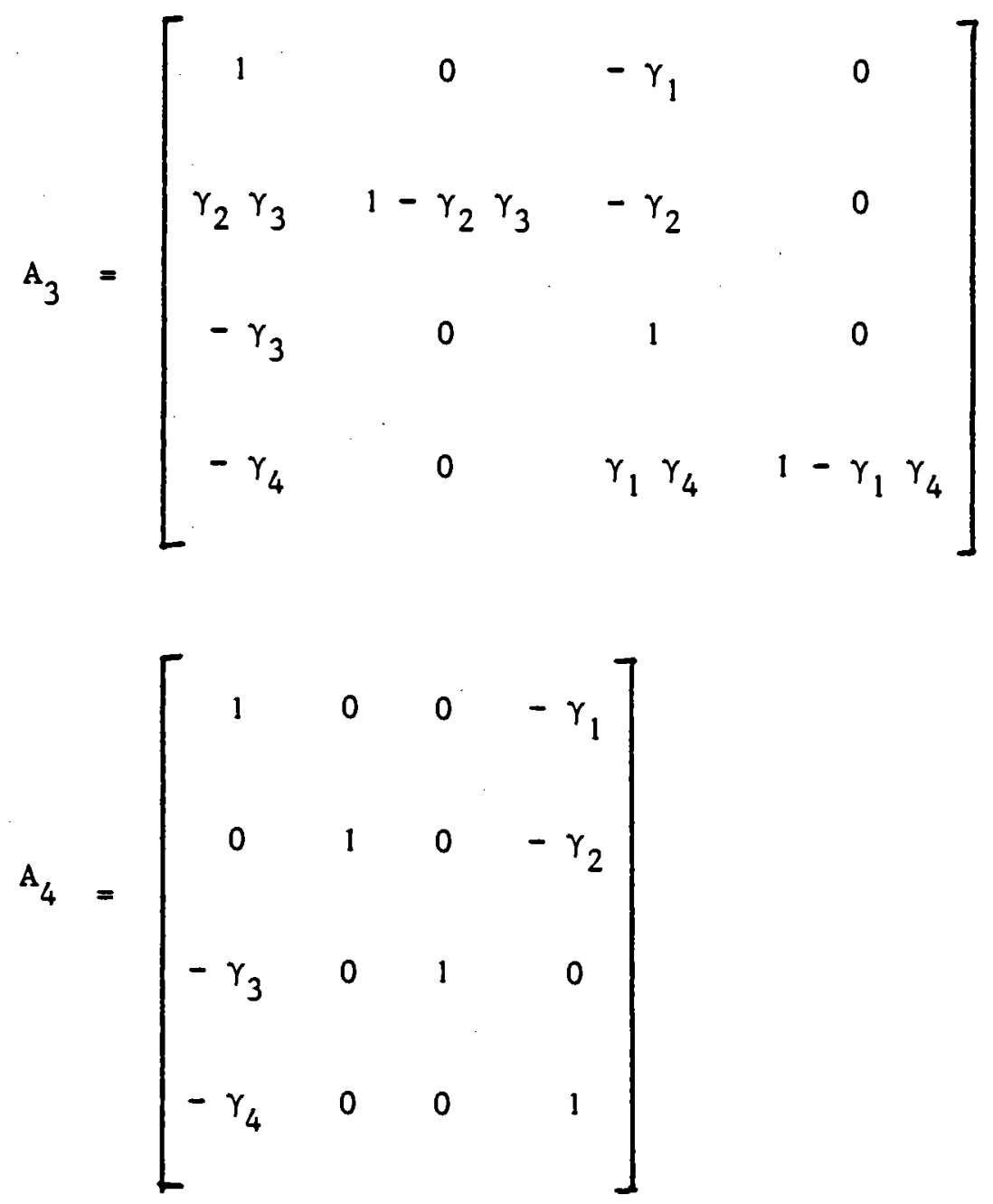

The point by point coincidence of these mappings on the intersection of the closures of the cones is obtained without any restriction. The global conservation of the boundaries of the cones requires $\left(1-\gamma_{1} \gamma_{4}\right)>0$ and $\left(1-\gamma_{2} \gamma_{3}\right)>0$. Under these conditions the determinants, det $A_{I}$ have the same sign if and only if $1-\gamma_{1} \gamma_{3}>0$ and $1-\gamma_{2} \gamma_{4}>0$. If all of these constraints are imposed, we obtain a well-defined reduced form. 
Example 2.2.:

We know from economic theory that there exists a correspondence of possible effective demands and any selection in this correspondence is a potential candidate. In GOURIEROUX and alii [3] we used the Clower effective demand; QUANDT [11] and ITO [6] use a different notion which is continuous on the boundaries of the regimes, and PORTES [10] has compared these two notions with a third one inspired from BENASSY [ 2].

We show below that the different notions of effective demands are related by piecewise linear mappings.

Consider two sets of linear demand and supply functions $D_{1 t}, S_{1 t}, D_{2 t}, S_{2 t}$ and $D_{1 t}^{\prime}, S_{1 t}^{\prime}, D_{2 t}^{\prime}, S_{2 t}^{r}$ inducing two sets of structural forms. They define the same model if and only if

$$
\begin{aligned}
& \min \left(D_{1 t}, S_{1 t}\right)=\min \left(D_{1 t}^{\prime}, S_{1 t}^{\prime}\right) \\
& \min \left(D_{2 t}, S_{2 t}\right)=\min \left(D_{2 t}^{\prime}, S_{2 t}^{\prime}\right)
\end{aligned}
$$

and

$$
\begin{aligned}
& D_{1 t}>S_{1 t} \Leftrightarrow D_{1 t}^{\prime}>S_{1 t}^{\prime} \\
& D_{2 t}>S_{2 t} \Leftrightarrow D_{2 t}^{\prime}>S_{2 t}^{\prime}
\end{aligned}
$$

These conditions joined to the linearity assumption imply within each regime $i$

$$
\begin{array}{ll}
D_{1 t}^{\prime}-S_{1 t}^{\prime}=\mu_{i}\left(D_{1 t}-S_{1 t}\right) & \mu_{i}>0 \\
D_{2 t}^{\prime}-S_{2 t}^{\prime}=v_{i}\left(D_{2 t}-S_{2 t}\right) & v_{i}>0
\end{array}
$$


Therefore this transformation is defined by eight parameters $\left(\mu_{1}, \mu_{2}, \mu_{3}, \mu_{4} ; \nu_{1}, \nu_{2}, \nu_{3}, \nu_{4}\right)$. In all cases, the assumptions of the theorem 2 are satisfied since $\mu_{i}>0, v_{i}>0$, $i=1, \ldots, 4$, and the coherency conditions are then fulfilled since the determinants of the mapping from $\left(D_{1 t}, S_{1 t}, D_{2 t}, S_{2 t}\right)$ to ( $D_{1 t}^{\prime}, S_{1 t}^{\prime}, D_{2 t}^{\prime}, S_{2 t}^{\prime}$ ), equal to $\mu_{i} \nu_{i}$, have the same sign; this means that this mapping is one to one.

The continuity requires in addition $\mu_{1}=\mu_{2}, \mu_{3}=\mu_{4}$, $v_{1}=v_{4}, v_{2}=v_{3}$

For example, the mapping from the vector $\left(D_{1 t}, S_{1 t}, D_{2 t}\right.$, $\mathrm{S}_{2 t}$ ) associated with the Clower effective demand to the vector $\left(D_{1 t}^{\prime}, S_{1 t}^{\prime}, D_{2 t}^{\prime}, S_{2 t}^{\prime}\right)$ associated with the Ito-Quandt effective demand is defined by (see PORTES [10 ]) :

$$
\begin{array}{ll}
\mu_{1}=\frac{1}{1-\gamma_{1} \gamma_{4}} & v_{1}=\frac{1}{1-\gamma_{2} \gamma_{3}} \\
\mu_{2}=1 & v_{2}=1 \\
\mu_{3}=\frac{1}{1-\gamma_{2} \gamma_{3}} & v_{3}=\frac{1}{1-\gamma_{1} \gamma_{4}} \\
\mu_{4}=1 & v_{4}=1
\end{array}
$$

The above continuity condition is not satisfied; it is not surprising since the Clower effective demand is discontinuous while the Ito-Quandt's one is not.

On the contrary, it is by a continuous mapping that the variables of the Ito-Quandt model are transformed into the variables of the Benassy model (see PORTES [10]). In that case we have : 


$$
-24-
$$

$$
\begin{array}{rlrl}
\mu_{1} & =\frac{1}{1-\gamma_{1} \gamma_{4}} & \nu_{1} & =\frac{1}{1-\gamma_{2} \gamma_{3}} \\
\mu_{2}=\frac{1}{1-\gamma_{1} \gamma_{4}} & \nu_{2} & =\frac{1}{1-\gamma_{1} \gamma_{4}} \\
\mu_{3}=\frac{1}{1-\gamma_{2} \gamma_{3}} & \nu_{3}=\frac{1}{1-\gamma_{1} \gamma_{4}} \\
\mu_{4}=\frac{1}{1-\gamma_{2} \gamma_{3}} & \nu_{4}=\frac{1}{1-\gamma_{2} \gamma_{3}}
\end{array}
$$

Here the continuity condition is satisfied. 


\section{3 - TYPE 3 HODELS : PIECELUISE AFFINE MAPPINGS ON CONES DEFINED BY ENDOGENOUS VARIABLES.}

A natural generalization of the problem considered in section 2 is the case in which the endogenous variables are transformed by affine mappings. Using the same notations as before, each affine mapping is defined on $C_{I}$ by :

$$
B_{I}(x)=A_{I}(x)+b_{I} \quad x \in \mathbb{R}^{n}
$$

where $A_{I}$ is an invertible linear mapping from $\mathbb{R}^{n}$ into $\mathbb{R}^{n}$ and $b_{I} \in \mathbb{R}^{n}$.

This kind of model appears when there are, at the same time, truncated variables (tobit or disequilibrium models) and dumy variables (probit models).

SCHMIDT [13] studied the "pure" simultaneous probit model, i.e. the case in which the endogenous variables are either untruncated or binary; mathematically this means that all the $A_{I}$ matrices are identical. The necessary and sufficient condition for coherency found by $S$ chmidt is essentially the recursivity of the model solved in terms of the untruncated variables.

In the following theorem we propose a sufficient condition for the general case.

Theorem 3 :

Under the same assumptions as in theorem 2, if

${ }^{b}{ }_{I} \in A_{I}\left(x \mid a_{j} x=0, j=1, \ldots, p\right) \quad \forall I$, then the mapping $f$ defined by 
$-26-$

$f(x)=\sum_{I} B_{I}(x) \mathbb{1}_{C_{I}}(x)=\sum_{I} A_{I}(x) \mathbb{D}_{C_{I}}(x)+\sum_{I} b_{I} \mathbb{1}_{C_{I}}(x)$ is interbible if and only if the determinants aet $A_{I}$ have the same sign.

Example 3.1.:

Consider a slight generalization of a model due to HECKMAN [4]

$$
\begin{aligned}
& \left\{\begin{array}{l}
y_{1 t}=\gamma_{1} y_{2 t}+\delta_{1}^{\prime} x_{1 t}+\mu_{1}+u_{1 t} \\
y_{2 t}=\gamma_{2} y_{1 t}+\delta_{2}^{\prime} x_{2 t}+\mu_{2}+u_{2 t}
\end{array} \quad \text { if } y_{2 t} \geqslant 0\right.
\end{aligned}
$$

The model can be rewritten as :

$$
\left[\begin{array}{cc}
1 & -\gamma_{1} \\
-\gamma_{2} & 1
\end{array}\right]\left[\begin{array}{l}
y_{1 t} \\
y_{2 t}
\end{array}\right]-\left[\begin{array}{l}
\mu_{1} \\
\mu_{2}
\end{array}\right]=\left[\begin{array}{ll}
\delta_{1}^{\prime} x_{1 t} \\
\delta_{2}^{\prime} x_{2 t}
\end{array}\right]+\left[\begin{array}{l}
u_{1 t} \\
u_{2 t}
\end{array}\right]
$$

if $y_{2 t} \geqslant 0$

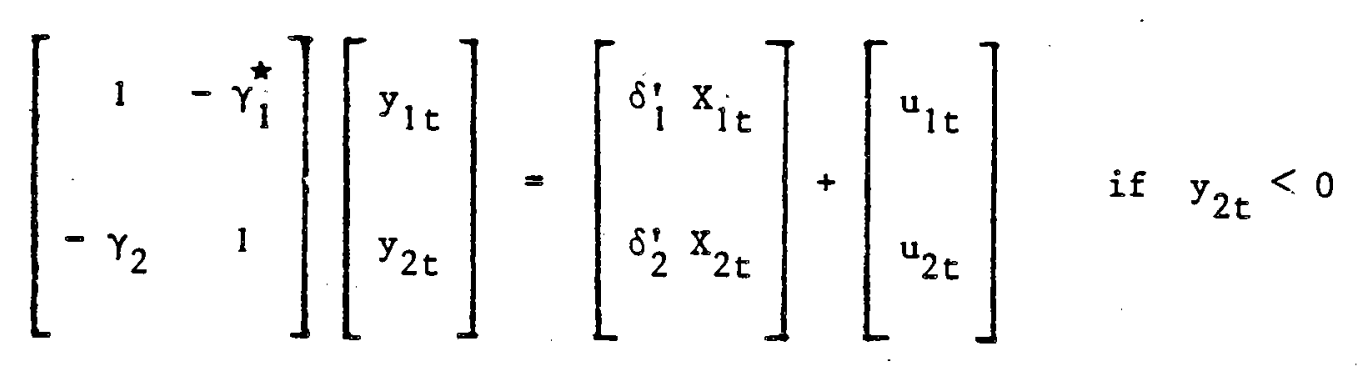


We have two regimes defined on the cones

$$
\begin{aligned}
& c_{1}=\left\{y_{t}=\left(y_{1 t}, y_{2 t}\right): a_{1} y_{t} \geqslant 0\right\} \quad \text { with } a_{1} y_{t}=y_{2 t} \\
& c_{2}=\left\{y_{t}=\left(y_{1 t}, y_{2 t}\right): a_{1} y_{t}<0\right\}
\end{aligned}
$$

The affine mappings are defined by :

$$
\begin{aligned}
& A_{i}=\left[\begin{array}{rr}
1 & -\gamma_{1} \\
-\gamma_{2} & 1
\end{array}\right] \quad b_{1}=-\left[\begin{array}{l}
\mu_{1} \\
\mu_{2}
\end{array}\right] \\
& A_{2}=\left[\begin{array}{rr}
1 & -\gamma_{1} \\
-\gamma_{2} & 1
\end{array}\right] \\
& b_{2}=\left[\begin{array}{l}
0 \\
0
\end{array}\right]
\end{aligned}
$$

The conditions $b_{I} \in A_{I}\left(x \mid a_{j} x=0, j=1, \ldots, p\right) \forall I$, are simply :

$$
\begin{aligned}
& \mathrm{b}_{1}=-\left[\begin{array}{l}
\mu_{1} \\
\mu_{2}
\end{array}\right]=\left[\begin{array}{l}
1 \\
\text { co-linear with } \\
0
\end{array}\right]=\left[\begin{array}{c}
1 \\
-\gamma_{2}
\end{array}\right] \\
& \text { or } \mu_{2}+\mu_{1} \gamma_{2}=0
\end{aligned}
$$


The determinants of $A_{1}$ and $A_{2}$ have the same sign iff $\left(1-\gamma_{1} \gamma_{2}\right)\left(1-\gamma_{1}^{\star} \gamma_{2}\right)>0$.

In this simple two dimensional case, it is easily seen that the condition $\mu_{2}+\mu_{1} \gamma_{2}=0$ is also necessary. The latter condition is also identical to the recursivity condition (see SCHMIDT [13]) in the "pure" probit case, i.e. when $\gamma_{1}=\gamma_{1}^{\star}$. 


\section{4 - TYPE 4 NODELS : CONTINUOUS PIECELISE AFFINE MAPPINGS ON BANOS DEFINED BY ENOUGENOUS VARIABLES.}

We have seen in theorems 1, 2, and 3 that the condition on determinants is an invertibility condition if we have $p \leqslant n$ independent linear forms defining $2^{\mathrm{P}}$ cones. In appendix 4 we give a counterexample showing that this result breaks down if there are $p>n$ linear forms.

In this section we shall see that we still have an invertibility theorem if $\mathbb{R}^{n}$ is partitioned in $q$ bands, $q$ being any integer (even greater than $2^{\mathrm{n}}$ ).

Let us denote by $a, a$ non-null linear form and by $k_{i}$ $(i=1, \ldots, q-1) q-1$ different numbers in increasing order.

We define $q$ bands in $\mathbb{R}^{n}$ by :

$c_{1}=\left\{x \mid a x \leqslant k_{1}\right\}$

$c_{i}=\left\{x \mid k_{i-1}<a x \leqslant k_{i}\right\}$

$c_{q}=\left\{x \mid a x>k_{q-1}\right\} \quad i=2, \ldots, q-1$

With each band $C_{i}$ we associate an affine mapping $B_{i}$, from $\mathbb{R}^{\mathbf{n}}$ into $\mathbb{R}^{\mathbf{n}}$, defined by
$B_{i}(x)=A_{i}(x)+b_{i}$
( $A_{i}$ invertible). 
We have the following result :

\section{Theorem 4:}

Assuming that the mapping $f$ defined by :

$f(x)=\sum_{i=1}^{q} A_{i}(x) \mathbb{1}_{C_{i}}(x)+\sum_{i=1}^{q} b_{i} \cdot \mathbb{1}_{C_{i}}(x)$

is continuous, $f$ is invertible if and only if the determinants, $\operatorname{det} A_{i}$, have the same sign.

\section{Example 4.1.:}

Let us consider a single market disequilibrium model where the systematic part of the supply function has two possible forms, one being a quantity constraint $k_{t}$ (for example an upper limit imposed by the Central Bank).

$$
\begin{aligned}
& D_{t}=\gamma_{1} P_{t}+\delta_{1}^{\prime} x_{1 t}+u_{1 t} \\
& s_{t}=\min \left(\gamma_{2} P_{t}+\delta_{2}^{\prime} x_{2 t}, k_{t}\right)+u_{2 t} . \\
& P_{t}-P_{t-1}=\lambda_{1}\left(D_{t}-s_{t}\right) \quad \text { if } D_{t} \geqslant s_{t} \\
& P_{t}-P_{t-1}=\lambda_{2}\left(D_{t}-s_{t}\right) \quad \text { if } D_{t}<s_{t},\left(\lambda_{1}>0, \lambda_{2}>0\right) \\
& Q_{t}=\min \left(D_{t}, S_{t}\right) .
\end{aligned}
$$


Replacing $P_{t}$ in terms of $D_{t}, S_{t}$ in the demand and supply equations, we obtain a piecewise affine mapping giving ( $u_{1 t}, u_{2 t}$ ) from $\left(D_{t}, S_{t}\right)$. As easily seen, this mapping is continuous. In order to define the different regimes we have to use the numbers :

$$
\begin{aligned}
& \alpha_{1 t}=\frac{k_{t}-\delta_{2}^{\prime} x_{2 t}-\gamma_{2} P_{t-1}}{\gamma_{2} \lambda_{1}} \\
& \alpha_{2 t}=\frac{k_{t}-\delta_{2}^{\prime} x_{2 t}-\gamma_{2} P_{t-1}}{\gamma_{2} \lambda_{2}}=\alpha_{1 t} \frac{\lambda_{1}}{\lambda_{2}}
\end{aligned}
$$

which have the same sign.

If $\alpha_{1 t}>0$ (and $\alpha_{2 t}>0$ ), we have three regimes defined on the following three bands of the space $\left(D_{t}, s_{t}\right)$ :

$$
\begin{aligned}
& c_{1}=\left\{D_{t}-s_{t}>\alpha_{1 t}\right\} \cap\left\{D_{t}-s_{t}>0\right\}=\left\{D_{t}-s_{t}>\alpha_{1 t}\right\} \\
& c_{2}=\left\{0<D_{t}-s_{t} \leqslant \alpha_{1 t}\right\} \\
& c_{3}=\left\{D_{t}-s_{t} \leqslant 0\right\} \cap\left\{D_{t}-s_{t} \leqslant \alpha_{2 t}\right\}=\left\{D_{t}-s_{t} \leqslant 0\right\}
\end{aligned}
$$$$
\text { If } \left.a_{1 t}<0 \text { ( and } \alpha_{2 t}<0\right) \text {, we also have three regimes }
$$

defined on

$$
\begin{aligned}
& C_{1}^{\prime}=\left\{D_{t}-s_{t}>\alpha_{1 t}\right\} \cap\left\{D_{t}-s_{t}>0\right\}=\left\{D_{t}-s_{t}>0\right\} \\
& c_{2}^{\prime}=\left\{\alpha_{2 t}<D_{t}-s_{t} \leqslant 0\right\} \\
& C_{3}^{\prime}=\left\{D_{t}-s_{t} \leqslant 0\right\} \cap\left\{D_{t}-s_{t} \leqslant \alpha_{2 t}\right\}=\left\{D_{t}-s_{t} \leqslant \alpha_{2 t}\right\}
\end{aligned}
$$

The different matrices $A_{i}$ are given below : 


\begin{tabular}{|c|c|c|}
\hline Band & Matrix & Determinants \\
\hline$c_{1}$ and $c_{1}^{\prime}$ & {$\left[\begin{array}{cc}1-\gamma_{1} \lambda_{1} & \gamma_{1} \lambda_{1} \\
0 & 1\end{array}\right]$} & $1-\gamma_{1} \lambda_{1}$ \\
\hline$c_{2}$ & {$\left[\begin{array}{cc}1-\gamma_{1} \lambda_{1} & \gamma_{1} \lambda_{1} \\
-\gamma_{2} \lambda_{1} & 1+\gamma_{2} \lambda_{1}\end{array}\right]$} & $1+\lambda_{1}\left(\gamma_{2}-\gamma_{1}\right)$ \\
\hline$C_{2}^{\prime}$ & {$\left[\begin{array}{cc}1-\gamma_{1} \lambda_{2} & \gamma_{1} \lambda_{2} \\
0 & 1\end{array}\right]$} & $1-\gamma_{1} \lambda_{2}$ \\
\hline$c_{3}$ and $C_{3}^{8}$ & {$\left[\begin{array}{cc}-\gamma_{1} \lambda_{2} & \gamma_{1} \lambda_{2} \\
-\gamma_{2} \lambda_{2} & 1+\gamma_{2} \lambda_{2}\end{array}\right]$} & $1+\lambda_{2}\left(\gamma_{2}-\gamma_{1}\right)$ \\
\hline
\end{tabular}

The coherency conditions are in particular fulfilled under the usual conditions :

$$
\gamma_{1}<0, \gamma_{2}>0, \lambda_{1}>0, \lambda_{2}>0
$$

Remark :

One may wonder whether theorem 4 is still valid if we do not assume that $f$ is continuous but if we only assume :

$$
B_{i-1}\left(\bar{C}_{i-1} \cap \bar{C}_{i}\right)=B_{i}\left(\bar{C}_{i-1} \cap \bar{C}_{i}\right) \quad i=2, \ldots, q
$$

(where $\bar{C}_{i}$ is the closure of $C_{i}$ ).

Unfortunately, in this case, the condition on the determinants is neither sufficient nor necessary (see appendix 4 for counterexamples). 
CONCLLOING REMARKS

The approach of non linear modelling by piecewise linear models with endogenously switching regimes, which seems to be powerful and flexible, probably deserves specific developments.

In this paper we solved the first problem raised by this approach, namely the coherency problem. The next problem which requires a systematic study is the identifiability question. The maximum likelihood techniques of estimation and testing are then readily implementable; however the non differentiability of these models necessitates a careful study of their asymptotic properties. 
[1] : AMEMIYA, T., "Multivariate Regression and Simultaneous Equation Models when the Dependant Variables are Truncated Normal", Econometrica, 42, (1974), 999-1012.

[2] : BENASSY, J.P., "Effective Demand, Quantity

Decision Theory", Scandinavian Journal of Economics.

[ 3] : GOURIEROUX, C., J.J. LAFFONT and A. MONFORT, "Disequilibrium Econometrics in Simultaneous Equations Systems", Ecole Polytechnique, D.P. $n^{\circ}$ A 1691177,1977 , to appear in Econometrica.

[4] : HECKRAN, J.J., "Dummy Endogenous Variables in a Simultaneous Equation System", Econometrica, 46, (1978), 931-960.

[5] : GOLDFELD, S.M. and R.E. QUANDT, "Estimation of a Disequilibrium Model and the Value of Information", Journal of Econometrics, 3 , 1975, 325-348.

[6] : ITO, T., "Methods of Estimation for Two-Market Disequilibrium Models", RIAS, W.P. ${ }^{\circ}$ 9, Harvard, 1977.

[ 7$]$ : LAFFONT, J.J. and A. MONFORT, "Econométrie des Modèles d'Equilibre avec Rationnement", Annales de 1'INSEE, 24, (1976). 
[8]: LEE, Lung-Fei, "Estimation of Limited Dependent Variable Models by Two-Stage Methods", Unpublished Doctoral Dissertation, University of Rochester, 1976.

[9] : MADdALA, G.S., "Selectivity Problems in Longitudinal Data", Annales de 1'INSEE, 30-31, 1978, 423-450.

[10]: PORTES, R., "Effective Demand and Spillovers in Empirical Two-Market Disequilibrium Models", Discussion Paper $\mathrm{N}^{\circ} 595$, Harvard Institute of Economic Research, 1977.

[11] : QUANDT, R., "Maximum Likelihood Estimation in Disequilibrium Models", Research Paper $n^{\circ}$ 198, Princeton University, 1976.

[12]: SAMELSON, H. R.M. THRALL and O. WESLER, "A Particular Theorem for Euclidian n Space", Proceeding of the American Mathematical Society, 9, (1958), 805-807.

[13]: SCHMDT, P., "Constraints on the Parameters in Simultaneous Tobit and Probit Models", 1978, Michigan State University. 


\section{APPENDIX 1 : PROOF OF THEOREM 1}

Our results are derived from a theorem proved by Samelson and alii [12] which gives a necessary and sufficient condition for a linear space to be partitioned in cones.

\section{First step :}

Let $E$ be the linear space $\mathbb{R}^{\mathrm{n}}$. Let $\left(\xi_{1}, \ldots, \xi_{\mathrm{n}}\right.$, $\left.n_{1}, \ldots, n_{n}\right)$ be $2 n$ vectors of $E$ such that any set of $n$ vectors $\left(\alpha_{1}, \ldots, \alpha_{n}\right)$ where $\alpha_{i}=\xi_{i}$ or $\eta_{i}$ is a basis of $\mathbb{R}^{n}$, and let I be a subset of $\{1,2, \ldots, n\}$.

Let $C_{I}$ be the positive cone generated by $\left(\alpha_{1}, \ldots, \alpha_{n}\right)$ where $\alpha_{i}=\xi_{i}$ if $i \in I$ and $\alpha_{i}=\eta_{i}$ if $i \notin I$, i.e.,

$$
C_{I}^{\star}=\left\{x \in E: x=\sum_{i=1}^{n} x_{i} \alpha_{i}, x_{i} \geqslant 0, i=1, \ldots, n\right\}
$$

We say that the $2^{n}$ cones $C_{I}^{\star}$ form a partition of $E$, if : $E=\underset{I}{U} C_{I}^{\star}$ and $\dot{C}_{I}^{\star} \cap \dot{C}_{J}^{\star}=\emptyset \forall I \neq J$, where $\dot{C}_{I}^{\star}$ is the interior of $C_{I}^{\star}$

In order to illustrate these definitions we give in the following figure an example of such a partition in $\mathbb{R}^{2}$. 


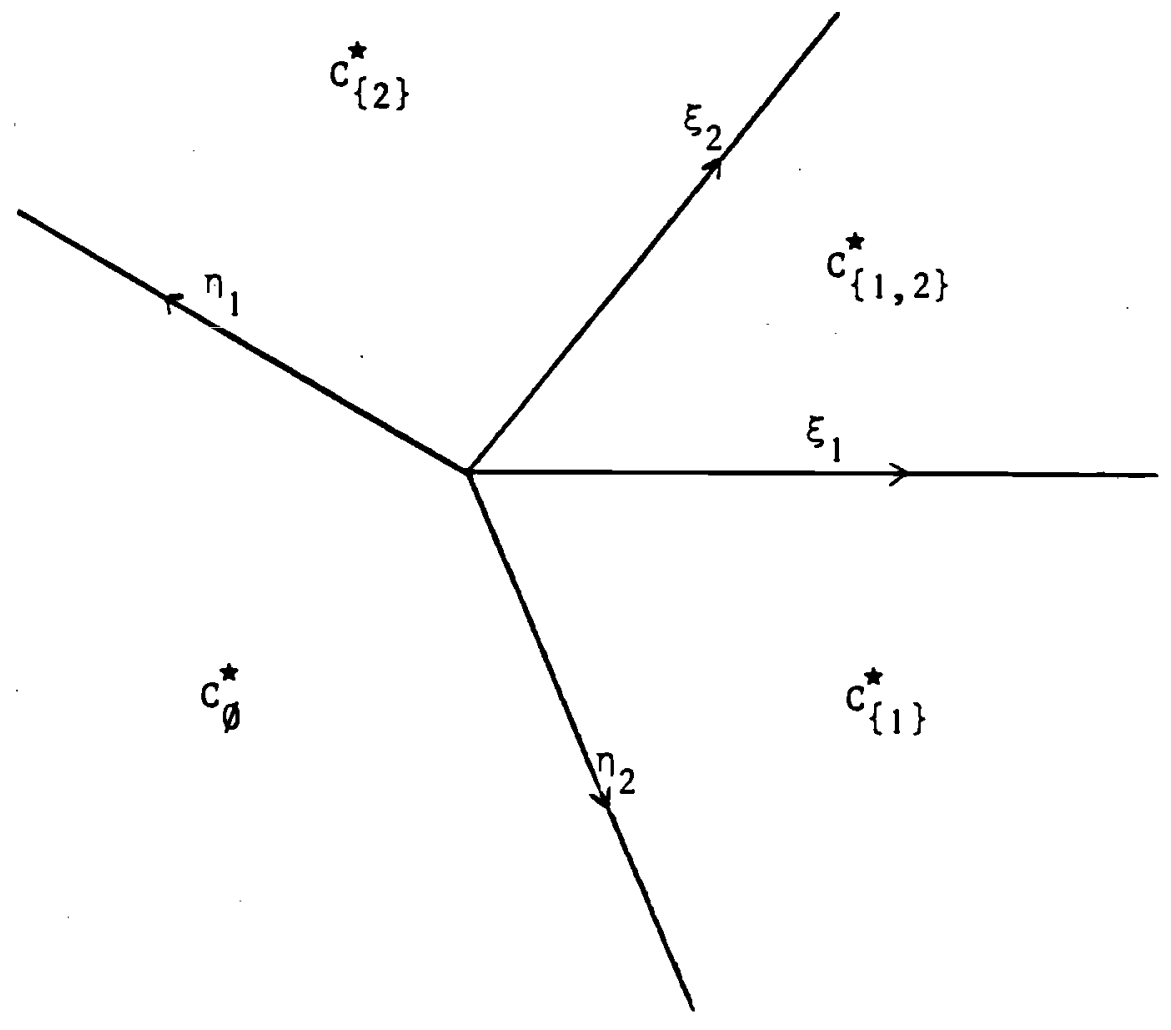

Lemma 1 :

A necessary and sufficient condition for the cones $C_{I}^{\star}$ to form a partition of $E$ is that the matrix $\Gamma$ of the vectors $\left(-n_{1}, \ldots,-n_{n}\right)$ in the basis $\left(\xi_{1}, \ldots, \xi_{n}\right)$ have all its principal minors positive.

Proof :

See $[12]$ 
We denote by $\Gamma^{(I)}, I \neq\{1,2, \ldots, n\}$, the matrix obtained from $\Gamma$ by deleting the lines and columns with index $i \in I$; $\Gamma^{(I)}$ is a $n-|I|$ by $n-|I|$ matrix, where $|I|$ is the number of elements in $I$. The condition of the theorem can be rewritten, $\forall I, \operatorname{det} \Gamma^{(I)}>0$.

\section{$\underline{\text { Second step : }}$}

Before considering the proof of theorem 1 , it is convenient to first solve the following special case.

Let us denote by $\left\{e_{1}, \ldots, e_{n}\right\}$ an orthonormal basis of $\mathbb{R}^{\mathrm{n}}$. For every subset $I$ included in $\{1,2, \ldots, n\}$, let $C_{I}$ be the orthant of $\mathbb{R}^{\mathrm{n}}$ defined as :

$$
\begin{aligned}
C_{I}=\left\{x / x=\sum_{i=1}^{n} x_{i} e_{i}\right. & \text { with } x_{i} \geqslant 0 \quad \forall i \in I \\
& \text { and } \left.x_{i}<0 \quad \forall i \notin I\right\}
\end{aligned}
$$

There are $2^{\mathrm{n}}$ such orthants. Let us associate with each orthant an invertible linear mapping $A_{I}$ from $\mathbb{R}^{n}$ into $\mathbb{R}^{n}$. We question the invertibility of the mapping $f=\sum_{I} A_{I} \mathbb{d}_{I}$.

\section{Lerma 2 :}

Suppose that the mapping $f=\sum_{I} A_{I} \mathbb{1}_{C_{I}}$ is continuous from $\mathbb{R}^{\mathrm{n}}$ into $\mathbb{R}^{\mathrm{n}}$. A necessary and sufficient condition for $f$ to be 
invertible is that all the determinants $\operatorname{det} A_{I}, I \subset\{1,2, \ldots, n\}$ have the same sign.

Proof :

$$
\text { Let us define: } \begin{aligned}
\xi_{i} & =A_{\{1, \ldots, n\}}\left(e_{i}\right) \\
\eta_{i} & =A_{\emptyset}\left(-e_{i}\right)
\end{aligned}
$$

- From the continuity of $f$, we derive that the system $\left(\alpha_{1}, \ldots, \alpha_{n}\right)$ where $\alpha_{i}=\xi_{i}$ if $i \in I$ and $\alpha_{i}=\eta_{i}$ if $i \notin I$ is the image by $A_{I}$ of the system $\left(e_{1}^{\star}, \ldots, e_{n}^{\star}\right)$ where $e_{i}^{\star}=e_{i}$ if $i \in I$ and $e_{i}^{\star}=-e_{i}$ if $i \notin I$. Since $A_{I}$ is invertible, $\left(\alpha_{1}, \ldots, \alpha_{n}\right)$ is a basis for any $I$.

$f$ is invertible if and only if the closure of the cones $f\left(C_{I}\right)=A_{I}\left(C_{I}\right)$ form a partition of $\mathbb{R}^{n}$. The closure of $A_{I}\left(C_{I}\right)$, denoted by $\overline{A_{I}\left(C_{I}\right)}$, is the positive cone generated by $\left(\alpha_{1}, \ldots, \alpha_{n}\right)$.

From lemma 1, a necessary and sufficient condition for $f$ to be invertible is that the trix $\Gamma$ of the system $\left(-n_{1}, \ldots,-n_{n}\right)$ in the basis $\left(\xi_{1}, \ldots, \xi_{n}\right)$ have all $i t s$ principal minors positive. Since $\forall i: A_{\emptyset} A_{\{1, \ldots, n\}}^{-1}\left(\xi_{i}\right)=-\eta_{i}$, the matrix $\Gamma$ is equal to $A_{0} A_{\{1, \ldots, n\}}^{-1}$ 
The matrix $\Gamma^{(I)}$, obtained from $\Gamma$ by deleting the lines and columns with index $i \in I$, has the same determinant as the matrix of the system $\left(\beta_{1}, \ldots, \beta_{n}\right)$ with $\beta_{i}=\xi_{i}$ if $i \in I$ and $\beta_{i}=-\eta_{i}$ if $i \notin I$ in the basis $\left(\xi_{1}, \ldots, \xi_{n}\right)$; this matrix is equal to $A_{I} A_{\{1, \ldots, n\}}^{-1}$

Therefore : f invertible

$\Leftrightarrow \operatorname{det} \Gamma^{(I)}=\operatorname{det}\left[\mathrm{A}_{I} \mathrm{~A}_{\{1, \ldots, \mathrm{n}\}}^{-1}\right]>0 \quad \forall I \neq\{1, \ldots, \mathrm{n}\}$

$\Leftrightarrow \operatorname{det} A_{I}$ has the same sign as $\operatorname{det} A_{\{1, \ldots, n\}} \forall I \neq\{1, \ldots, n\}$

$\Leftrightarrow$ all the deteminants det $A_{I}$ have the same sign $\forall I$.

$$
\text { Q.E.D. }
$$

\section{Third step :}

Consider now the general context of section 1, where the cones $\mathrm{C}_{\mathrm{I}}$ are defined by $\mathrm{n}$ independent linear forms $a_{1}, \ldots, a_{n}$ :

$$
C_{I}=\left\{x / x \in \mathbb{R}^{n}, a_{i} x \geqslant 0 \quad \forall i \in I \text { and } a_{i} x<0 \forall i \notin I\right\},
$$

and $f=\sum_{I} A_{I} \mathbb{Z}_{I}$

By choosing an appropriate basis, we can assume that $a_{i} x=x_{i}$ $\left(i^{\text {th }}\right.$ coordinate of $\left.x\right), i=1, \ldots, n$. 
If $P$ is the matrix of this change of basis, we know from lemma 2 that a necessary and sufficient condition for the invertibility of $f$ is : " $\operatorname{det}\left(P^{-1} A_{I} P\right)$ of the same sign for any $I$ ".

This condition is equivalent to " $\operatorname{det} A_{I}$ of the same sign for any I".

$$
\text { Q.E.D. }
$$


APPENDIX 2 : PROOFS OF THEOREMS 2 ANO 3.

\section{Proof of theorem 2.}

\section{First step :}

Let us first consider the case in which there are $p=n$

independent linear forms. By choosing an appropriate basis we can assume that these linear forms $a_{i}, i=1, \ldots, n$ are such that :

$a_{i} x=x_{i}\left(i^{\text {th }}\right.$ coordinate of $\left.x\right) ;$ this choice implies that the $2^{n}$ cones $C_{I}$ are the orthants associated with the canonical basis $\left(e_{1}, \ldots, e_{n}\right)$.

The function $f=\sum_{I} A_{I} \mathbb{1}_{C_{I}}$ is discontinuous. Let us consider the function $f^{\star}=\sum_{I} A_{I}^{\star} \square_{C}$, where $A_{I}^{\star}$ is defined by ;

$\forall i=1, \ldots, n: A_{I}^{\star}\left(e_{i}\right)=\frac{A_{I}\left(e_{i}\right)}{\left\|A_{I}\left(e_{i}\right)\right\|}$

The second assumption of theorem 2 implies that $f^{\star}$ is continuous.

Moreover the images of the cones $C_{I}$ by $f$ and $f^{\star}$ are the same for any $I$, therefore $f$ is invertible if and only if $f^{\star}$ is invertible. Applying theorem 1 to $f^{\star}$, the coherency condition is " $\operatorname{det} A_{I}^{\star}$ of the same sign for any $I "$. Since $A_{I}^{\star}$ is obtained from $A_{I}$ by multiplying each column by a positive number, $\operatorname{det} A_{I}$ and $\operatorname{det} A_{I}$ have the same sign and the coherency condition is also " det $A_{I}$ of the same sign for any I ". 


\section{Second step :}

Let us consider the general case $p \leqslant n$. Each of the $2^{p}$ cones $C_{I}, I \subset\{1, \ldots, p\}$ is an union of orthants $C_{J}$, $J \subset\{1, \ldots, n\}$. On all the orthants $C_{J}$ corresponding to the same $C_{I}$, the restrictions $\AA_{J}$, of $f$ are the same and are equal to that of $A_{I}$ - f can be written :

$$
E=\sum_{I} A_{I}{ }_{C_{I}}=\sum_{J} \AA_{J} \underset{\tilde{C}_{J}}{1}
$$

From the first step the coherency condition is " $\operatorname{det} \tilde{A}_{J}$ of the same sign $\forall J \subset\{1, \ldots, n\} "$. Since the determinant of $\AA_{J}$ is equal to the determinant of the associated $A_{I}$ the coherency condition is also " $\operatorname{det} A_{I}$ of the same sign".

$$
\text { Q.E.D. }
$$

\section{Proof of theorem 3 .}

Onder the assumptions of theorem 3 the images of $C_{I}$ by $B_{I}$. and $A_{I}$ are the same. Therefore $f=\sum_{I} B_{I} \mathbb{d}_{C_{I}}$ is invertible if and only if $\sum_{I} A_{I} \mathbb{1}_{C_{I}}$ is invertible and the result follows from theorem 2. 
APPENDIX 3 : PROOF OF THEOREII 4.

By choosing appropriate bases on the domain space and on the range space we can have: $a=(1,0, \ldots, 0)$ and $A_{i}\left\{x \mid x \in \mathbb{R}^{n}, x_{1}=0\right\}$ $=\left\{y \mid y \in \mathbb{R}^{\mathbb{n}}, y_{1}=0\right\}$ for $i=1, \ldots, q$

From now, we denote $\left\{x_{1}=0\right\}=\left\{x \mid x \in \mathbb{R}^{n}, x_{1}=0\right\}$

The latter condition can be rewritten:

$\forall x_{2}, \ldots, x_{n} \quad A_{i}\left[\begin{array}{c}0 \\ x_{2} \\ \vdots \\ x_{n}\end{array}\right] \quad=\left[\begin{array}{c}0 \\ y_{2} \\ \vdots \\ y_{n}\end{array}\right] \quad i=1, \ldots, q$ which implies that the first row of the $A_{i}$ 's is of the form:

$$
\left(\alpha_{i}, 0, \ldots, 0\right)
$$

Moreover the continuity of $f$ implies that the $A_{i}$ 's are identical on $\left\{x_{1}=0\right\}$; therefore the $A_{i}$ 's have the following structure:

$$
A_{i}=\left[\begin{array}{l|lll}
\alpha_{i} & 0 & \ldots & 0 \\
\hline & & M
\end{array}\right]
$$

where the $(n-1) \times(n-1)$ matrix $M$ is the same for all the $A_{i}$ 's.

Let $d_{i}$ be the number such that :

$$
\begin{aligned}
& B_{i}\left\{x_{1}=k_{i}\right\}=B_{i+1}\left\{x_{1}=k_{i}\right\}=\left\{y_{1}=d_{i}\right\} \quad i=1, \ldots, q \\
& \text { If } \alpha_{i}>0, \text { then } d_{i-1}<d_{i} \text { and } \\
B_{i}\left(C_{i}\right)= & B_{i}\left\{k_{i-1}<x_{1} \leqslant k_{i}\right\}=\left\{d_{i-1}<y_{1}<d_{i}\right\} \\
& \text { If } \alpha_{i}<0, \text { then } d_{i-1}>d_{i} \text { and } \\
B_{i}\left(C_{i}\right)= & B_{i}\left\{k_{i-1}<x_{1} \leqslant k_{i}\right\}=\left\{d_{i} \leqslant y_{1}<d_{i-1}\right\} \quad i=2, \ldots, q-1
\end{aligned}
$$


(For $i=1$ or $i=q$, we have similar results; for instance

$$
\begin{aligned}
& \text { if } \alpha_{1}>0 \quad B_{1}\left(c_{1}\right)=B_{1}\left\{x_{1} \leqslant k_{1}\right\}=\left\{y_{1} \leqslant d_{1}\right\} \\
& \text { if } \alpha_{1}<0 \quad B_{1}\left(c_{1}\right)=B_{1}\left\{x_{1} \leqslant k_{1}\right\}=\left\{y_{1} \geqslant d_{1}\right\}
\end{aligned}
$$

We are now able to show that $f$ is invertible if and only if the $\alpha_{i}$ 's have the same sign. The condition is necessary because if $\alpha_{i}$ and $\alpha_{i+1}$ have different signs, say $\alpha_{i}>0$ and $\alpha_{i+1}<0$, we sec that:

$$
B_{i}\left(C_{i}\right) \cap B_{i+1}\left(C_{i+1}\right)=\left\{d_{i-1}<y_{1} \leqslant d_{i}\right\} \cap\left\{d_{i+1} \leqslant y_{1}<d_{i}\right\}=\emptyset
$$

therefore, $f$ is not invertible.

The condition is sufficient because, if the $\alpha_{i}$ 's have the same sign, say $\alpha_{i}>0 \quad \forall i$, the bands

$$
\begin{array}{ll}
B_{i}\left(c_{i}\right)=\left\{d_{i-1}<y_{1} \leqslant d_{i}\right\} \quad \text { (with } i=1, \ldots, q+1 \text { and } \\
\left.d_{0}=-\infty, d_{q+1}=+\infty\right)
\end{array}
$$

define a partition in $\mathbb{R}^{\mathbf{n}}$.

To complete the proof we have only to note that the $\alpha_{i}$ 's have the same sign if and only if the determinants of the $A_{i}$ 's have the same sign, since $\operatorname{det} A_{i}=a_{i} \operatorname{det} M$

Q.E.D. 
1) The condition on the determinants is no longer valid if the cones are defined by more than $n$ linear forms (even in the continuous case).

Consider for instance the mapping $f$, from $\mathbb{R}^{2}$ into $\mathbb{R}^{2}$ defined

as :

$$
\mathbf{f}=\sum_{i=1}^{8} A_{i} C_{i}
$$

where the $A_{i}$ 's and the $C_{i}$ 's are given below:

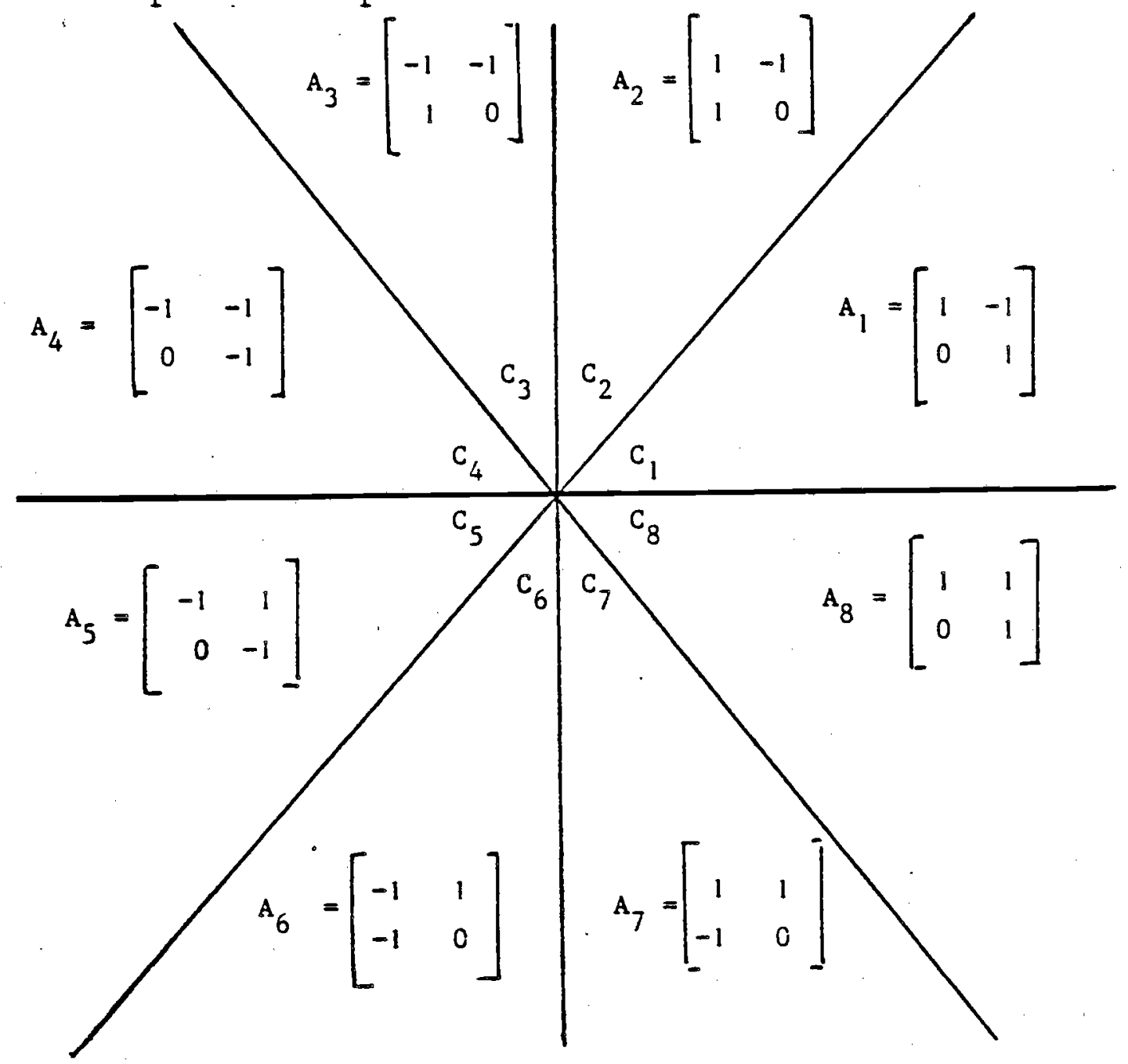


All the determinants are positive, $f$ is obviously continuous, however $f$ is not invertible since $f(x)=f(-x) \forall x \in \mathbb{R}^{2}$.

2) Theorem 4 is no longer valid if we only assume that :

$$
B_{i-1}\left(\bar{C}_{i-1} \cap \bar{C}_{i}\right)=B_{i}\left(\bar{C}_{i-1} \cap \bar{C}_{i}\right) \quad i=2, \ldots, q
$$

Consider the case $n=2$ and the following bands(or cones)

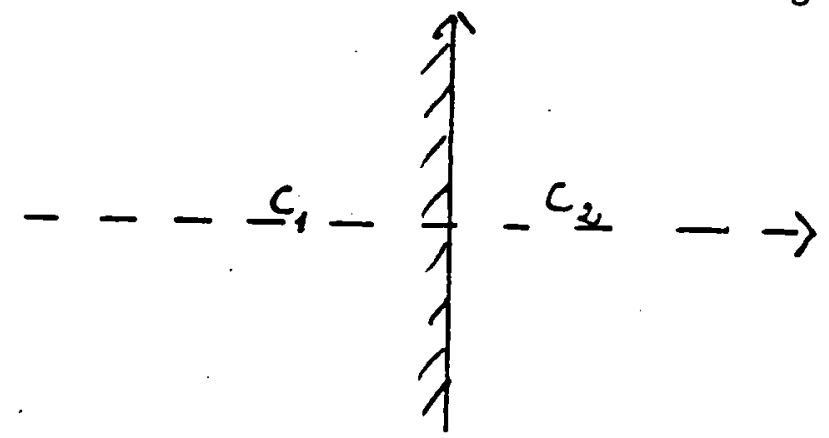

a) if $A_{1}=\left[\begin{array}{ll}1 & 0 \\ 0 & 1\end{array}\right]$ and $A_{2}=\left[\begin{array}{rr}1 & 0 \\ 0 & -1\end{array}\right]$

$f=\sum_{i=1}^{2} A_{i} \mathbb{C}_{C}$ is invertible but det $A_{1}$ and $\operatorname{det} A_{2}$ are not

of the same sign.

$$
\begin{aligned}
& \text { b) if } A_{1}=\left[\begin{array}{rr}
-1 & 0 \\
0 & -1
\end{array}\right] \text { and } A_{2}=\left[\begin{array}{ll}
1 & 0 \\
0 & 1
\end{array}\right] \\
& f=\underset{i=1}{2} A_{i} \quad \mathbb{C}_{i} \text { is not invertible, however } \operatorname{det} A_{1}=\operatorname{det} A_{2}=1
\end{aligned}
$$

